\title{
Primary Multidrug Resistant Tuberculosis and Utility of Line Probe Assay for Its Detection in Smear-Positive Sputum Samples in a Tertiary Care Hospital in South India
}

\author{
Fahmiya Leena Yacoob, Beena Philomina Jose, \\ Sarada Devi Karunakaran Lelitha, and Sreelatha Sreenivasan \\ Department of Microbiology, Government Medical College, Kozhikode, Kerala 673008, India \\ Correspondence should be addressed to Fahmiya Leena Yacoob; yfahmiya@gmail.com
}

Received 16 October 2015; Revised 5 January 2016; Accepted 2 March 2016

Academic Editor: Abhineet S. Sheoran

Copyright ( 2016 Fahmiya Leena Yacoob et al. This is an open access article distributed under the Creative Commons Attribution License, which permits unrestricted use, distribution, and reproduction in any medium, provided the original work is properly cited.

\begin{abstract}
In a high tuberculosis burdened country like India, rapid, cost-effective, and reliable diagnostic tools for tuberculosis are an urgent need of the hour to prevent inappropriate treatment strategies and further spread of resistance. This study aimed to estimate the proportion of new smear-positive tuberculosis cases with primary resistance to rifampicin and/or isoniazid as well as identify the common mutations associated with it. Sputum of 200 newly diagnosed smear-positive cases of $1+$ score and above was directly subjected to Line Probe Assay using the GenoType MTBDRplus assay kit. All samples were inoculated onto solid media and 61 samples were inoculated in automated liquid culture also. The Line Probe Assay gave hundred percent interpretable results with $2.5 \%$ of the study population showing resistant pattern. Only $1 \%$ of the cases were primary multidrug resistant tuberculosis and $1.5 \%$ showed isoniazid monoresistance. S531L and C15T were the most common genetic mutations seen for rifampicin and isoniazid resistance, respectively. $40 \%$ had absent $r p o B$ wild type 8 band indicating probable silent mutation after clinical correlation. The average turnaround time for Line Probe Assay was far less (3.8 days) as compared to solid and liquid cultures (35.6 days and 13.5 days, resp.).
\end{abstract}

\section{Introduction}

Tuberculosis (TB) earned the sobriquet "The Captain of All These Men of Death" during the 18th and 19th century for the morbidity and mortality it caused and now ranks alongside HIV as a leading cause of death worldwide [1]. India ranks first among the high TB burdened countries contributing to $24 \%$ of the estimated global incidence. It is also one of the three countries contributing to more than half of the global burden of multidrug resistant tuberculosis (MDR TB), the other two being China and the Russian Federation $[2,3]$.

Improved rapid diagnostic methods, vigilant monitoring of case reporting, and follow-up through national surveillance programs in these countries will have a global impact on the TB statistics. Although MDR TB is reported to be low with $2.2 \%$ among new cases and $15 \%$ among retreatment cases, due to the large population and high TB burden, it translates into a high number of actual cases (71,000 estimated MDR TB cases among notified pulmonary TB cases in 2014) [1]. Sputum smear microscopy remains the mainstay of diagnosis due to its simplicity, cost-effectiveness, and feasibility. However, molecular diagnostic techniques like GeneXpert, Polymerase Chain Reaction (PCR), and Line Probe Assays (LPAs) are being increasingly used recently. These newer techniques aid in the rapid diagnosis of drug resistance and thus prevent inappropriate treatment regimes, amplification, and spread of drug resistance in countries like India with high TB load. The World Health Organization (WHO) recommended a new policy to use LPAs for rapid screening of patients at risk of MDR TB in 2008 [4].

The LPA is a reverse hybridization technique wherein the patient's sample, after undergoing DNA extraction and amplification, is subjected to hybridization with membrane strips 
coated with complementary probes targeted against specific genes. Highly specific binding of complementary DNA strands is ensured by stringent conditions which result from the combination of buffer composition and a certain temperature. During hybridization, streptavidin-conjugated alkaline phosphatase is added which binds to the amplicon's biotin via the streptavidin moiety. Finally, the alkaline phosphatase transforms an added substrate into a dye which become visible on the membrane strips as a coloured precipitate. A template helps in the interpretation of the banding patterns obtained.

GenoType MTBDRplus assay (Hain Lifesciences, GmbH, Nehren, Germany) targets the $r p o B$ (coding for beta subunit of RNA polymerase), katG (coding for catalase peroxidase), and promoter region of inhA (coding for NADH enoyl ACP reductase) genes in both culture isolates and clinical samples. Thus, in addition to rifampicin resistance ( $r p o B$ gene), MTBDRplus assay aids in the detection of high level and low level resistance to isoniazid (INH) via the kat G gene and inhA gene, respectively. In India, as per the Revised National TB Control Programme (RNTCP), the definition of presumptive drug resistant $\mathrm{TB}$ (DR TB) cases includes all retreatment cases at diagnosis, any smear-positive cases during follow-up, contacts of confirmed DR TB cases, and HIV associated TB cases at diagnosis [5]. For drug susceptibility testing (DST), rapid molecular tests like LPA and CB-NAAT (cartridge based-nucleic acid amplification tests), if available, are the preferred DST methods for first-line drugs as per RNTCP protocol [5]. This study aimed at finding the proportion of cases with primary drug resistance in the study population and to assess the utility of LPA in our set-up in comparison to solid and automated liquid culture systems.

\section{Materials and Methods}

2.1. Specimen Collection and Storage. Early morning expectorated sputum samples of 200 new smear-positive cases of grade $1+$ and above were collected in sterile leakproof containers during the study period of one and a half years. All the samples were stored at a temperature of $2-8^{\circ} \mathrm{C}$ for not more than 4 days before decontamination.

\subsection{Specimen Processing}

2.2.1. Direct Smears. The specimens were stained by ZiehlNeelsen technique. The smears were graded as "scanty" for 1$10 \mathrm{AFB} / 100$ oil emulsion fields (OIF), "1+" for 10-99 AFB/100 OIF, “2+" for 1-9 AFB/OIF, and " $3+$ ” for $>10$ AFB/OIF. Smears graded "scanty" positive were excluded from this study.

2.2.2. Decontamination. It was done by $\mathrm{N}$-acetyl-L-cysteine(NALC-) $\mathrm{NaOH}$-sodium citrate method in which equal amounts of $4 \% \mathrm{NaOH}$ and $2.9 \%$ sodium citrate were mixed with NALC powder $(0.5 \mathrm{~g}$ NALC/ $100 \mathrm{~mL}$ of $\mathrm{NaOH}$-sodium citrate solution). This mixture was used within 24 hours of preparation. In a $50 \mathrm{~mL}$ plastic sterile conical bottom graduated centrifuge tube, specimen $\leq 10 \mathrm{~mL}$ and NALC solution were added in equal volume. The capped tubes were vortexed (about 5-20 seconds/tube) and after allowing it to stand for 15-20 minutes, the tubes were filled to $50 \mathrm{~mL}$ with sterile phosphate buffer solution at pH 6.8 and swirled by hand to mix. The specimens were then concentrated in a centrifuge at a speed of 3,000 g for 15 minutes and the supernatant fluid was carefully decanted from the pellet. The pellet sediments were resuspended with phosphate buffer ( $\mathrm{pH}$ 6.8) to achieve a final volume of $1 \mathrm{~mL}$.

2.2.3. Culture. All samples were subjected to solid culture on Lowenstein-Jensen (LJ) media. Sixty-one samples were inoculated into MB-Bact automated liquid culture media. This protocol was made to ensure that, in cases where sputum samples gave invalid/negative result with direct LPA, their culture isolates can be processed as per the manufacturer's instructions and subjected to LPA (GenoType MTBDRplus ver 2.0). However, none of the samples gave invalid results.

2.2.4. DNA Extraction and Amplification. The decontaminated samples were stored at $-20^{\circ} \mathrm{C}$ for not more than 5 days until DNA extraction was carried out. $500 \mu \mathrm{L}$ of the decontaminated specimen was taken in a $1.5 \mathrm{~mL}$ microfuge tube and centrifuged at 10,000 g for 15 minutes. The pellet was resuspended in $200 \mu \mathrm{L}$ of molecular biology grade water and incubated at $95^{\circ} \mathrm{C}$ for 20 minutes in a water bath. Then it was incubated for 15 minutes in an ultrasonic bath. It was spun down for 5 minutes at $14,000 \mathrm{~g}$ and $5 \mu \mathrm{L}$ of the supernatant was used directly for PCR.

After DNA extraction, each sample $(5 \mu \mathrm{L})$ was mixed with $10 \mu \mathrm{L}$ AM-A and $35 \mu \mathrm{L}$ AM-B (amplification mix provided with the kit) to make a final volume of $50 \mu \mathrm{L}$. Instead of the extracted DNA solution, $5 \mu \mathrm{L}$ of molecular biology grade water was added to the negative control. Amplification was done in thermal cycler as per the kit manual [6]. In case of delay, the amplification products were stored at $-20^{\circ} \mathrm{C}$ until further use.

2.2.5. Hybridization. The Hain protocol as per the manufacturer's instructions was followed.

Incubation was done in "TwinCubator" which is a semiautomatic washing and shaking device with a capacity to hold 12 samples at a time. Hybridization steps were performed using reagents provided with the kit. $20 \mu \mathrm{L}$ of Denaturation Solution (DEN) was dispensed in a corner of each of the wells used. The amplified products were then carefully added and mixed. After five minutes of incubation, hybridization buffer was added and a single strip was placed in each well with coated side facing up. Following incubation and washing steps, the diluted conjugate was added and kept for further incubation. Finally, the substrate was added and the strips were incubated protected from light. Washing steps with stringent and rinse wash solutions were performed as required by the kit protocol. After final wash step with distilled water, the strips were removed using tweezers and dried between 2 layers of absorbent paper. The developed sheets were pasted on the evaluation sheet provided with the kit in the designated fields by aligning the bands CC and AC with the respective lines. 
TABLE 1: Category I (DOTS) and category IV (DOTSplus) regimen advocated under RNTCP for new TB and MDR TB patients, respectively.

\begin{tabular}{lll}
\hline & Intensive phase & Continuation phase \\
\hline $\begin{array}{l}\text { Category I } \\
\text { (DOTS) }\end{array}$ & $\begin{array}{l}\text { Rifampicin + INH + ethambutol + pyrazinamide } \\
\text { (thrice a week for 2 months) }\end{array}$ & $\begin{array}{l}\text { Rifampicin + INH } \\
\text { (thrice a week for 4 months) }\end{array}$ \\
$\begin{array}{l}\text { Category IV } \\
\text { (DOTSplus) }\end{array}$ & $\begin{array}{l}\text { Kanamycin + ofloxacin (levofloxacin) + ethionamide + } \\
\text { pyrazinamide + ethambutol + cycloserine } \\
\text { (daily for 6-9 months) }\end{array}$ & $\begin{array}{l}\text { Ofloxacin (levofloxacin) }+ \text { ethionamide + ethambutol + } \\
\text { cycloserine } \\
\text { (daily for 18 months) }\end{array}$ \\
\hline
\end{tabular}

Each strip has a total of 27 reaction zones. The presence of wild type bands without a mutant band is interpreted as sensitive and the presence of a mutant band with or without the simultaneous absence of the corresponding wild type band is interpreted as resistant pattern.

All patients were registered under the RNTCP Unit of Government Medical College, Kozhikode, and they were on regular follow-up. All smear-positive patients were initiated on category I regimen of "directly observed treatment shortcourse" (DOTS) and those with confirmed MDR TB were initiated on category IV regimen (DOTSplus) (Table 1).

\section{Results}

A total of 200 new sputum smear-positive samples were processed and directly subjected to LPA. All the samples gave valid and interpretable results as indicated by the presence of all control loci bands. The negative controls showed the presence of conjugate control (CC) and amplification control (CC) bands only (Figure 1). All the samples showed presence of TUB band thereby suggesting infection with Mycobacterium tuberculosis complex.

Out of the 200 samples, $1 \%$ (2 samples) showed MDR TB pattern on LPA. An additional 1.5\% (3 samples) showed INH monoresistance in this study.

The two samples with MDR TB pattern on LPA were confirmed from an Intermediate Reference Laboratory (IRL) in Kerala by both LPA and phenotypic DST. Both the MDR TB isolates in this study showed absent $r p o B$ WT8 band and presence of $r p o B$ MUT3 band (Figures 2 and 3) which represents a mutation and amino acid change from serine to leucine associated with codon number 531 of the $r p o B$ gene.

A significant association was found between the presence of rifampicin resistance and INH resistance in this study as both the samples with rifampicin resistance had resistance to INH also.

Among the two MDR cases detected in this study, one patient had mutation in both the high level and low level INH resistance determining regions indicated by the presence of kat G MUT1 band corresponding to S315T1 mutation in codon 315 of katG gene and inhA MUT3A band corresponding to $\mathrm{T} 8 \mathrm{C}$ mutation in the -8 nucleic acid position of the inhA promoter region, respectively. Both the INH mutations showed heteroresistant pattern; that is, there was simultaneous presence of both mutant and wild type bands for the specified genes.

The other MDR sample showed only low level INH heteroresistance indicated by the presence of inhA MUT1

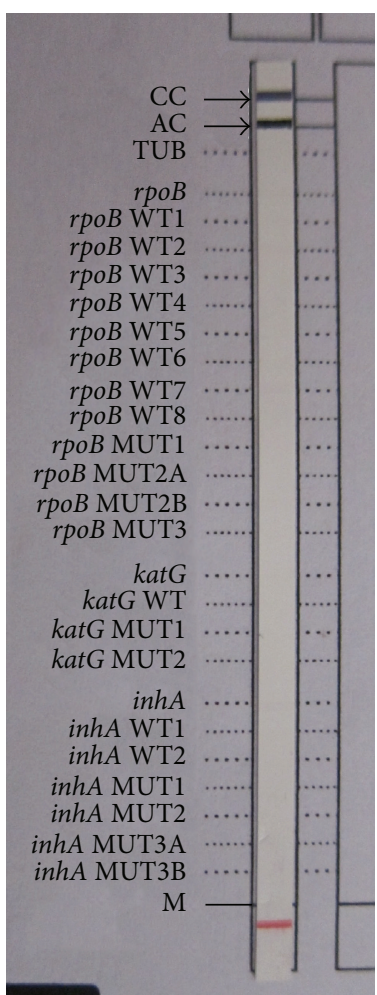

FIgURE 1: Negative control with CC and AC bands. The red band is reference marker to align the strip.

band corresponding to C15T mutation in the -15 nucleic acid position of the inhA promoter region.

Both the patients were diabetic and one of them was HIV positive. They were initiated on category IV regimen after confirmation of this study's findings by phenotypic DST.

Out of the 3 patients (1.5\%) with INH monoresistance, 2 patients had low level INH heteroresistance showing inhA MUT1 band corresponding to C15T mutation in the -15 nucleic acid position of the inhA promoter region with simultaneous presence of wild type bands (Figure 4). Both the patients improved under category I treatment regimen.

The third patient had similar mutation, C15T, indicating low level INH monoresistance but with absent wild type band (Figure 5). This patient had coexisting morbidities of diabetic kidney disease with bilateral hydroureteronephrosis and renal TB. She succumbed to death while on category I treatment. However the cause of the death cannot be completely attributed to INH resistance due to her otherwise poor health condition. 


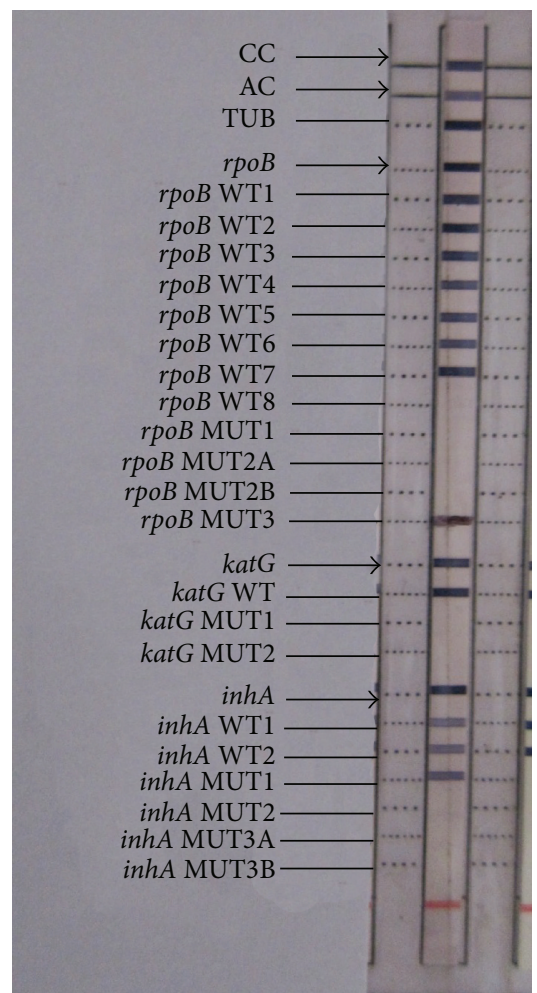

FIGURE 2: Patient with MDR TB showing presence of $r p o B$ MUT3 band and inhA MUT1 band along with the wild type bands indicating rifampicin resistance and INH heteroresistance, respectively.

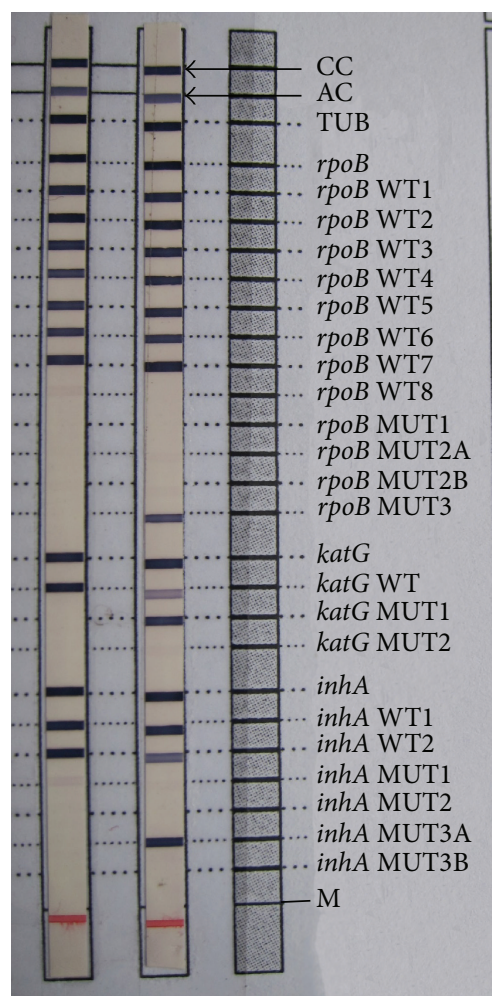

FIGURE 3: (From left to right) Lane 1: sensitive pattern. Lane 2: MDR TB patient with $r p o B$ MUT3 band indicating rifampicin resistance and $k a t G$ MUT1 band and inhA MUT3A band along with wild type band indicating INH heteroresistance. Lane 3: reference strip.

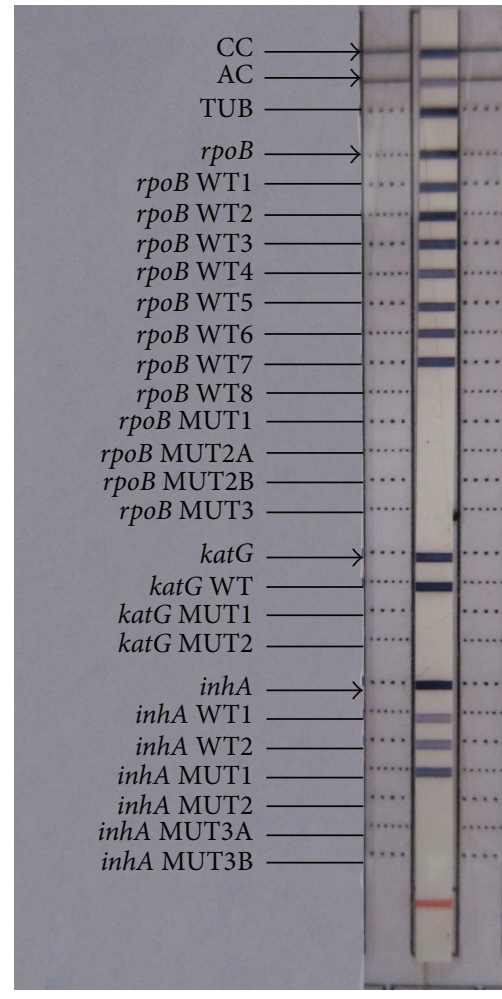

FIGURE 4: Patient with inhA MUT1 band along with wild type bands indicating low level INH heteroresistance.

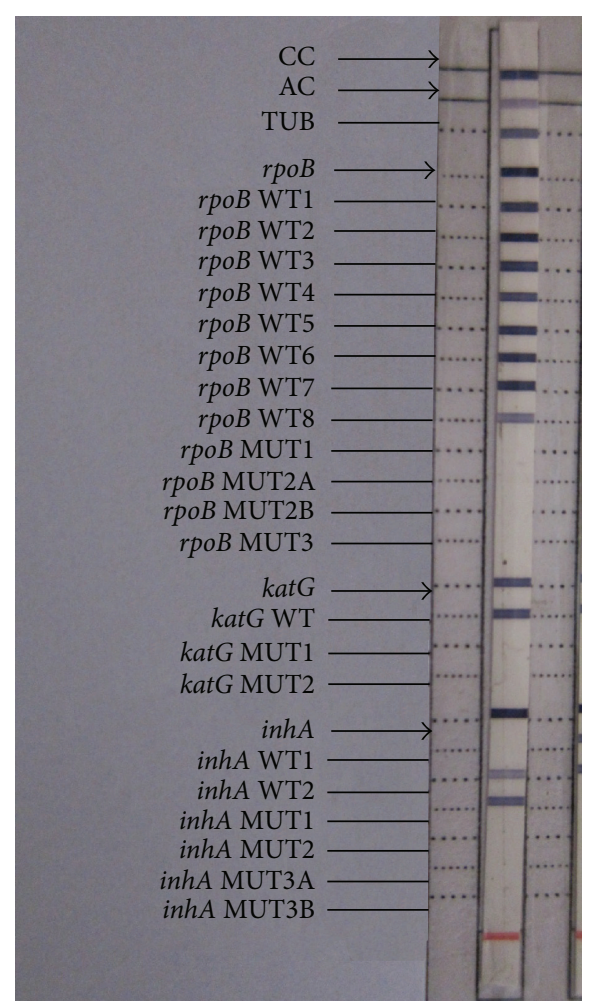

FIgURE 5: Patient showing low level INH monoresistance with absent inhA WT1 band and presence of inhA MUT1 band. 


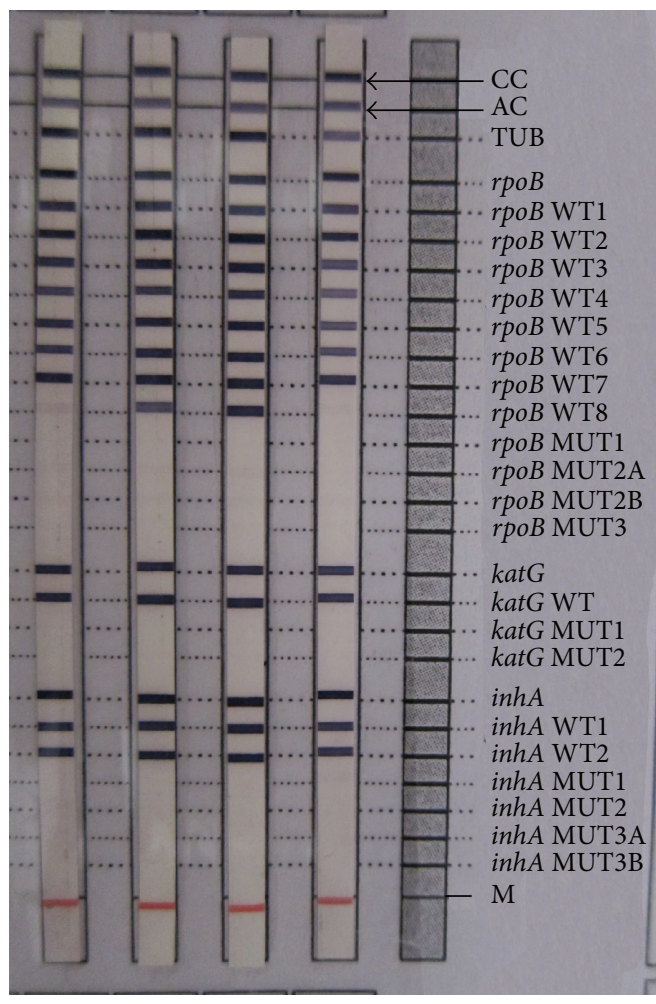

Figure 6: (From left to right) Lane 1: faint $r p o B$ WT8 band interpreted as sensitive pattern. Lanes 2 and 3: sensitive pattern. Lane 4: sensitive pattern with absent $r p o B$ WT8 band.

Another interesting observation in this study was the absence of $r p o B$ WT8 band in almost $40 \%$ of the samples (Figure 6). Faint band was taken as positive as per the manual along with the kit.

All the patients with only absent rpoB WT8 band responded to category I regimen with follow-up sputum smears being negative.

\subsection{Comparison of LPA and Solid and Automated Liquid} Culture. The isolation rate in LJ medium in the present study was $79 \%$ and that in MB-Bact was $91.8 \%$. In comparison, the LPA gave a valid result in all 200 samples.

In the present study, average detection time was 35.6 days by conventional solid culture (Range 17-50 days) and 13.5 days by automated MB-Bact system (Range 5-25 days). On the other hand, the average turnaround time of LPA from receipt of sample to the reporting of result was 3.8 days (Range 2-8 days).

3.2. Epidemiological Characteristics of Study Population. Of the 200 samples, 162 (81\%) were from males with a femaleto-male ratio of $0.23: 1$.

The age and sex distribution of the study population is depicted in Figure 7.

In this study, $4.5 \%$ of the study population were positive for HIV which is consistent with the RNTCP finding stating that the TB epidemic in India continues to be predominantly

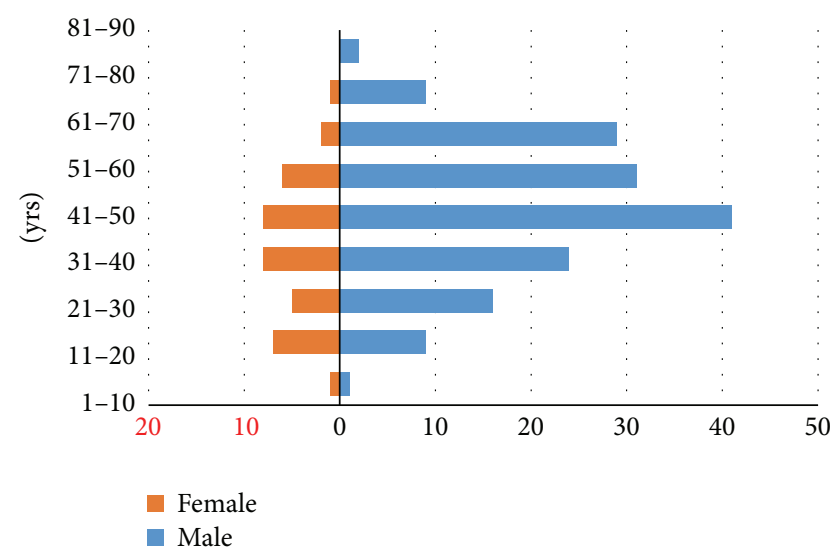

Figure 7: Age and gender distribution of the study population. The majority of the patients are of the age group between 41 and 50 with male preponderance.

TABLE 2: Relationship between diabetes mellitus and sputum smear grade. Higher smear grades were observed in patients who were diabetic. ( $P$ value: 0.022$)$.

\begin{tabular}{lcccc}
\hline Diabetes & \multicolumn{3}{c}{ Smear Grade } & Total \\
& $1+$ & $2+$ & $3+$ & \\
\hline Present & $11(22.4 \%)$ & $19(38.8 \%)$ & $19(38.8 \%)$ & 49 \\
Absent & $67(44.4 \%)$ & $45(29.8 \%)$ & $39(25.8 \%)$ & 151 \\
\hline Total & $78(39 \%)$ & $64(32 \%)$ & $58(29 \%)$ & 200 \\
\hline
\end{tabular}

driven by the pool of HIV negative TB infected individuals [7].

Among the tuberculosis patients with diabetes, $77.6 \%$ had higher smear grades of $2+$ and $3+$. This study showed a significant association between diabetes mellitus (DM) and smear grade with a higher bacillary load being observed in diabetic patients (Table 2). DM has received recent recognition as a risk factor for TB and epidemiological studies have elucidated an association between DM and the development of TB [8].

\section{Discussion}

This study was conducted in a tertiary care hospital in Northern Kerala, Government Medical College, Kozhikode, to assess the proportion of primary MDR TB among new sputum smear-positive cases and the utility of LPA for its rapid detection. This study also aimed to find the most common genetic mutations associated with rifampicin and INH resistance in the study population.

In India, MDR TB among new cases are estimated at 2$3 \%$ [7]. This study showed low rates (1\%) of MDR TB among new sputum positive pulmonary TB patients similar to other Indian studies $[9,10]$. The prevalence of INH monoresistance ranging from $1 \%$ to $7 \%$ has been reported in various Indian studies [11, 12] and our study population showed $1.5 \%$ INH monoresistance.

The most common mutation associated with rifampicin resistance was S531L mutation in the $r p o B$ region consistent with the findings of various other studies [12-15]. 
There are publications $[16,17]$ stating that rifampicin resistance can be a surrogate marker for MDR TB since more than $90 \%$ of rifampicin-resistant isolates are also isoniazid resistant. But, a systematic review [18] concluded that rapid tests for rifampicin resistance alone cannot accurately predict rifampicin resistance or MDR TB in areas with low prevalence of rifampicin resistance. However, in areas with high prevalence of rifampicin resistance and MDR TB, these tests may be of value in MDR TB management strategy.

In case of INH resistance, heteroresistant patterns were observed more commonly in our study. Heteroresistance is a preliminary stage towards full resistance which is difficult to detect using conventional DST methods. However, studies on heteroresistance have shown that phenotypic DST results corresponded to the mutated, that is, resistant, organism [19, 20]. The development of heteroresistance has been explained in previous studies as occurring in two ways: the coexistence of two subpopulations (sensitive and resistant) of the same $M$. tuberculosis strain or two separate strains of sensitive and resistant $M$. tuberculosis occurring simultaneously in the same sample. These mechanisms were linked to the clinical entities: new cases, treatment failures, and relapses.

So as explained by Tolani et al. [21], heteroresistance could have arisen due to transmission of both susceptible and resistant bacterial populations from drug resistant patients to previously untreated cases or the presence of exclusively sensitive bacteria at onset which gradually develop resistance during therapy, with incomplete elimination of the sensitive population, and would result in phenotypic resistance but with both forms remaining detectable genotypically.

In this study, the common mutation associated with INH resistance was $\mathrm{C} 15 \mathrm{~T}$ in the -15 nucleic acid position of the promoter region of $i n h A$ gene. The prevalence of mutations in the kat $G$ and $i n h A$ genes seems to vary widely in different geographic locations as reported in various studies conducted in different countries $[12,22,23]$. In a study by Maurya and et al. [24], the most prominent mutations in $r p o B, k a t G$, and inhA genes were S531L, S315T1, and C15T, respectively, similar to this study.

INH resistance occurs due to substitutions in the catalaseperoxidase $k a t G$ gene in more than three-quarters of cases and, more rarely, due to mutation in inhA (less than 10\%) or the $a p h C$ gene. The predominant mode of acquisition of resistance via kat $G$ alteration is the selection of particular mutation, that is, the transversion of codon 315 AGC-ACC (Ser-Thr) that decreases the catalase activity, but maintains a certain level of the peroxidase activity of the enzyme in viable INH-resistant organisms [25].

In this study, the 2 patients with low level INH heteroresistance improved under category I regimen. According to another study [26], inhA mutations were not associated with unfavorable treatment outcome. However, the study states that inhA mutations do increase the risk of relapse with treatment regimens that contain only INH and ethambutol in the continuation phase as the probability of survival of bacteria with an inhA mutation inside macrophages is higher than for the katG315 mutant strains because they still have full catalase-peroxidase expression. Whether the increased risk of relapse, in particular for inhA mutations, also exists with the World Health Organization- (WHO-) recommended 6month regimen (2RHEZ/4RH) remains to be studied [26].

The absence of WT8 band in almost $40 \%$ of the study population is a matter which needs clarification through further studies. The kit manual mentions that the absence of WT8 band in the $r p o B$ region may be attributed to a silent mutation L533P (Leu (CTG) to Pro (CCG)) [6]. One study [27] reported that the change in codon 533 or 515 does not likely correlate with the rifampicin-resistant phenotype. Another study by Raveendran et al. [28] states that such discrepancy may be encountered in this assay and in such cases phenotypic results are considered confirmatory.

However, all the patients in this study with absent WT8 band improved under category I regimen. Furthermore, all these patients were new smear-positive cases with no suspicion of drug resistance. Thus, if deletion of WT8 band is the only indication of rifampicin resistance and there is no mutant probe, it would be advisable to continue the patient on rifampicin therapy till phenotypic sensitivity report is available.

Various studies report different turnaround time for LPA ranging from a period within 24 hours to a period of 5 days $[13,28,29]$. The variation is probably due to lesser frequency of samples being received per day and doing the assay batchwise, only when sufficient samples are present to be done in a single run.

Nonetheless, the use of LPA significantly reduces the turnaround time as compared to solid and automated liquid culture systems. In an average of 3.8 days, the result along with drug sensitivity of rifampicin and INH could be obtained by LPA. Hence, the time to diagnosis and administration of proper treatment is effectively reduced. This has a major impact on reducing the spread of MDR TB and its efficient management.

Different studies have already demonstrated the feasibility of MTBDRplus assay as an effective tool in early detection of MDR TB and have good concordance with phenotypic DST. The performance of LPAs has been adequately validated in direct testing of sputum smear-positive specimens and on isolates of $M$. tuberculosis complex grown from smearnegative and smear-positive specimens. However, the direct use of LPA is not recommended on smear-negative-clinical specimens [30].

\section{Conclusions}

The proportion of primary pulmonary MDR TB as well as INH monoresistance was relatively low in the present study group with the most common mutation in the S531L region of $r p o B$ gene for rifampicin resistance and C15T in the inh $A$ promoter region for INH resistance. This study emphasizes that the implementation of LPA with its rapid turnaround time and high specificity and sensitivity for detection of MDR TB directly from sputum smear-positive samples will be an unprecedented milestone in the diagnosis, treatment, and management of MDR TB. However, the LPA cannot completely replace phenotypic culture methods, as evident by the above results where silent mutations may complicate the clinical interpretation and phenotypic DST is advocated 
in such cases. A limitation of this study was the inability to perform phenotypic DST due to time and staff constraints.

In conclusion, the LPA definitely aids earlier diagnosis and proper management of MDR TB, although, as with other molecular tests, judicious decision to supplement LPA with conventional methods should be used wherever necessary.

\section{Competing Interests}

The authors declare that there are no competing interests regarding the publication of this paper.

\section{Acknowledgments}

Dr. Fahmiya would like to thank Dr. Rema Devi S., Former Head, Department of Microbiology, for her permission to conduct the study. Dr. Fahmiya also extends heartfelt gratitude to Dr. Biju M. George, Dr. Shabana O., Dr. Jaishid Ahdal, and Ms. Sreeja for their valuable inputs and suggestions during this study.

\section{References}

[1] World Health Organization, Global Tuberculosis Report 2015, World Health Organization, Geneva, Switzerland, 2015.

[2] Regional Office for South-East Asia, Tuberculosis in South East Asia Region, Annual TB Report 2015, World Health Organization-SEAR, New Delhi, India, 2015.

[3] Drug-Resistant TB, "Surveillance and response, supplement," Global TB Report, World Health Organization, Geneva, Switzerland, 2014.

[4] WHO, "Molecular line probe assays for rapid screening of patients at risk of MDR-TB," Policy Statement, WHO, 2008, http://www.who.int/tb/features_archive/policy_statement.pdf.

[5] TB India, Revised National Tuberculosis Control Programme Annual Status Report, Government of India, New Delhi, India, 2014.

[6] GenoType MTBDRplus-Hain Lifescience Ver 2.0, Instructions for use, IFU-304A-02, 2012.

[7] Training Module for Medical Practitioners, Central TB Division, Directorate General of Health Services, Ministry of Health and Family Welfare, Central TB Division, Revised National Tuberculosis Control Programme, 2010.

[8] C. Y. Jeon and M. B. Murray, "Diabetes mellitus increases the risk of active tuberculosis: a systematic review of 13 observational studies," PLoS Medicine, vol. 5, no. 7, pp. 1091-1101, 2008.

[9] S. K. Sharma, G. Kaushik, B. Jha et al., "Prevalence of multidrugresistant tuberculosis among newly diagnosed cases of sputumpositive pulmonary tuberculosis," Indian Journal of Medical Research, vol. 133, no. 3, pp. 308-311, 2011.

[10] M. R. Joseph, C. T. Shoby, G. R. Amma, L. S. Chauhan, and C. N. Paramasivan, "Surveillance of anti-tuberculosis drug resistance in Ernakulam District, Kerala State, South India," International Journal of Tuberculosis and Lung Disease, vol. 11, no. 4, pp. 443449, 2007.

[11] D. T. B. D'Souza, N. F. Mistry, T. S. Vira et al., "High levels of multidrug resistant tuberculosis in new and treatmentfailure patients from the revised national tuberculosis control programme in an urban metropolis (Mumbai) in Western India," BMC Public Health, vol. 9, article 211, 2009.
[12] S. Sharma, M. Madan, C. Agrawal, and A. K. Asthana, "Genotype MTBDR plus assay for molecular detection of rifampicin and isoniazid resistance in Mycobacterium tuberculosis," Indian Journal of Pathology and Microbiology, vol. 57, no. 3, pp. 423426, 2014.

[13] N. Mohan, P. B. Chandrasekhar, I. J. Padmaja, N. Raizada, P. S. Rao, and B. S. Kumar, "Genotype MTBDRplus line probe assay for rapid and direct detection of rifampicin and isoniazid resistance in Mycobacterium tuberculosis complex from sputum samples," Journal of Dr. NTR University of Health Sciences, vol. 3, pp. 23-27, 2014.

[14] D. Hillemann, S. Rüsch-Gerdes, and E. Richter, "Evaluation of the GenoType MTBDRplus assay for rifampin and isoniazid susceptibility testing of Mycobacterium tuberculosis strains and clinical specimens," Journal of Clinical Microbiology, vol. 45, no. 8, pp. 2635-2640, 2007.

[15] M. A. L. Lingala, A. Srikantam, S. Jain, K. V. S. M. Rao, and P. V. Ranganadha Rao, "Clinical and geographical profiles of $r p o B$ gene mutations in Mycobacterium tuberculosis isolates from Hyderabad and Koraput in India," Journal of Microbiology and Antimicrobials, vol. 2, no. 2, pp. 13-18, 2010.

[16] P. Deepa, K. L. Therese, and H. N. Madhavan, "Detection and characterization of mutations in rifampicin resistant Mycobacterium tuberculosis clinical isolates by DNA sequencing," Indian Journal of Tuberculosis, vol. 52, pp. 132-136, 2005.

[17] F. A. Drobniewski and S. M. Wilson, "The rapid diagnosis of isoniazid and rifampicin resistance in Mycobacterium tuberculosis-a molecular story," Journal of Medical Microbiology, vol. 47, no. 3, pp. 189-196, 1998.

[18] M. Arentz, B. Sorensen, D. J. Horne, and J. L. Walson, "Systematic review of the performance of rapid rifampicin resistance testing for drug-resistant tuberculosis," PLoS ONE, vol. 8, no. 10, Article ID e76533, 2013.

[19] P. Kumar, V. Balooni, B. K. Sharma, V. Kapil, K. S. Sachdeva, and S. Singh, "High degree of multi-drug resistance and heteroresistance in pulmonary TB patients from Punjab state of India," Tuberculosis, vol. 94, no. 1, pp. 73-80, 2014.

[20] S. Hofmann-Thiel, J. Van Ingen, K. Feldmann et al., "Mechanisms of heteroresistance to isoniazid and rifampin of Mycobacterium tuberculosis in Tashkent, Uzbekistan," European Respiratory Journal, vol. 33, no. 2, pp. 368-374, 2009.

[21] M. P. Tolani, D. T. B. D’souza, and N. F. Mistry, "Drug resistance mutations and heteroresistance detected using the GenoType MTBDRplus assay and their implication for treatment outcomes in patients from Mumbai, India," BMC Infectious Diseases, vol. 12, article 9, 2012.

[22] I. Mokrousov, O. Narvskaya, T. Otten, E. Limeschenko, L. Steklova, and B. Vyshnevskiy, "High prevalence of KatG Ser315Thr substitution among isoniazid-resistant Mycobacterium tuberculosis clinical isolates from northwestern Russia, 1996 to 2001," Antimicrobial Agents and Chemotherapy, vol. 46, no. 5, pp. 1417-1424, 2002.

[23] A. Van Rie, R. Warren, I. Mshanga et al., "Analysis for a limited number of gene codons can predict drug resistance of Mycobacterium tuberculosis in a high-incidence community," Journal of Clinical Microbiology, vol. 39, no. 2, pp. 636-641, 2001.

[24] A. K. Maurya, J. Umrao, A. K. Singh, S. Kant, R. A. Kushwaha, and T. N. Dhole, "Evaluation of GenoType ${ }^{\circledR}$ MTBDRplus assay for rapid detection of drug susceptibility testing of multi-drug resistance tuberculosis in Northern India," Indian Journal of Pathology and Microbiology, vol. 56, no. 2, pp. 139-143, 2013. 
[25] M. Veluchamy, R. Madhavan, S. Narayanan, and L. Rajesh, "KatG gene as a surrogate molecular marker leading to cause drug resistance in Mycobacterium tuberculosis isolates," American Journal of Infectious Diseases and Microbiology, vol. 1, no. 5, pp. 86-91, 2013.

[26] M. N. T. Huyen, F. G. J. Cobelens, T. N. Buu et al., "Epidemiology of isoniazid resistance mutations and their effect on tuberculosis treatment outcomes," Antimicrobial Agents and Chemotherapy, vol. 57, no. 8, pp. 3620-3627, 2013.

[27] H. Ohno, H. Koga, S. Kohno, T. Tashiro, and K. Hara, "Relationship between rifampin MICs for and $r p o B$ mutations of Mycobacterium tuberculosis strains isolated in Japan," Antimicrobial Agents and Chemotherapy, vol. 40, no. 4, pp. 1053-1056, 1996.

[28] R. Raveendran, C. Wattal, J. K. Oberoi, N. Goel, S. Datta, and K. J. Prasad, "Utility of GenoType MTBDRplus assay in rapid diagnosis of multidrug resistant tuberculosis at a tertiary care centre in India," Indian Journal of Medical Microbiology, vol. 30, no. 1, pp. 58-63, 2012.

[29] R. Anek-vorapong, C. Sinthuwattanawibool, L. J. Podewils et al., "Validation of the GenoType ${ }^{\circledR}$ MTBDRplus assay for detection of MDR-TB in a public health laboratory in Thailand," $B M C$ Infectious Diseases, vol. 10, article 123, 2010.

[30] S. K. Patra and A. Jain, "Molecular diagnosis of multi drug resistant tuberculosis," International Journal of Biomedical and Advance Research, vol. 3, no. 5, pp. 273-280, 2012. 


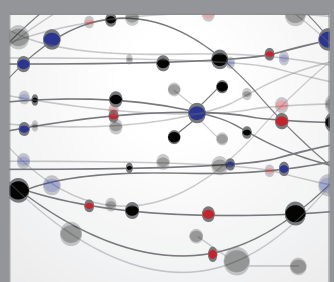

The Scientific World Journal
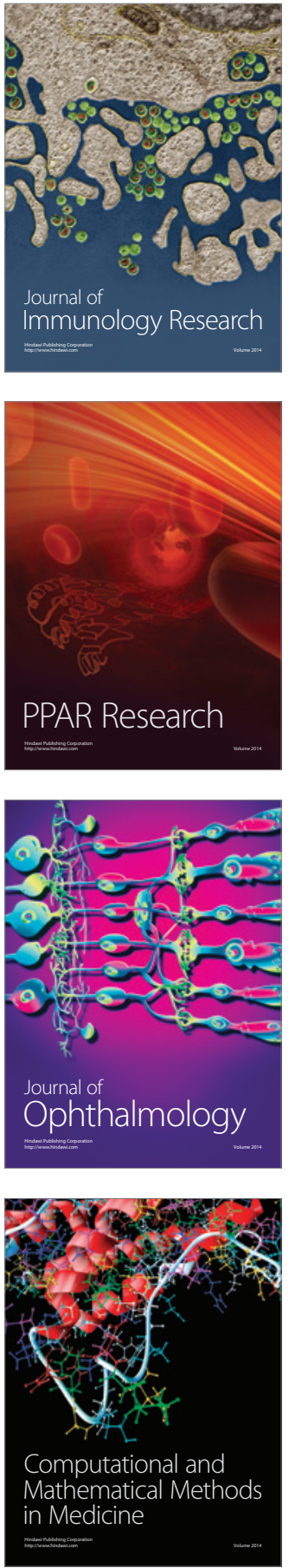

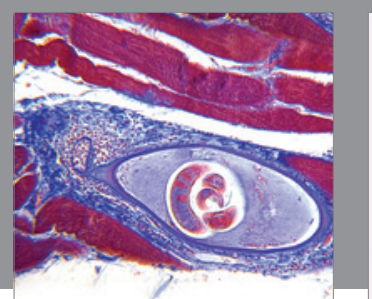

Gastroenterology Research and Practice

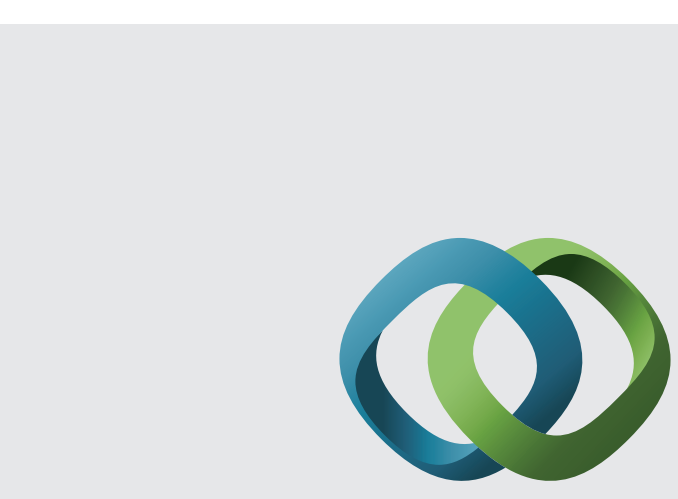

\section{Hindawi}

Submit your manuscripts at

http://www.hindawi.com
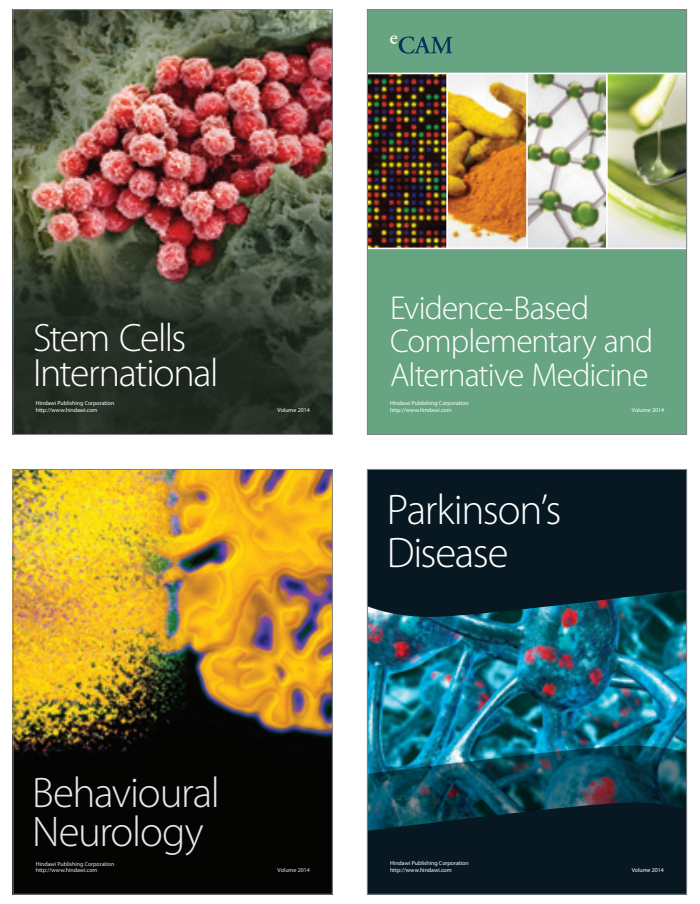
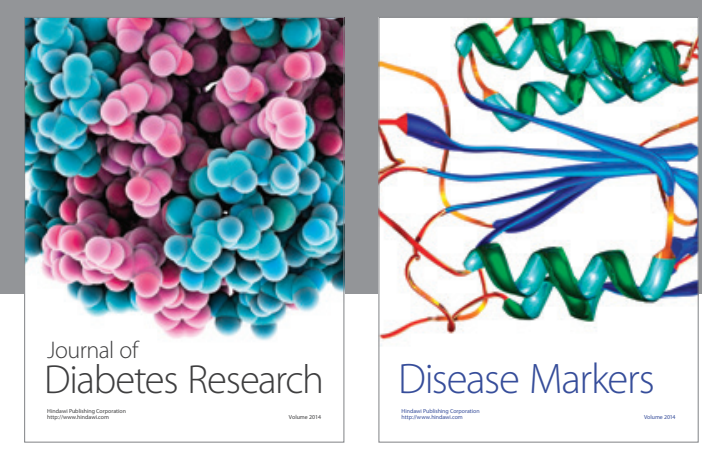

Disease Markers
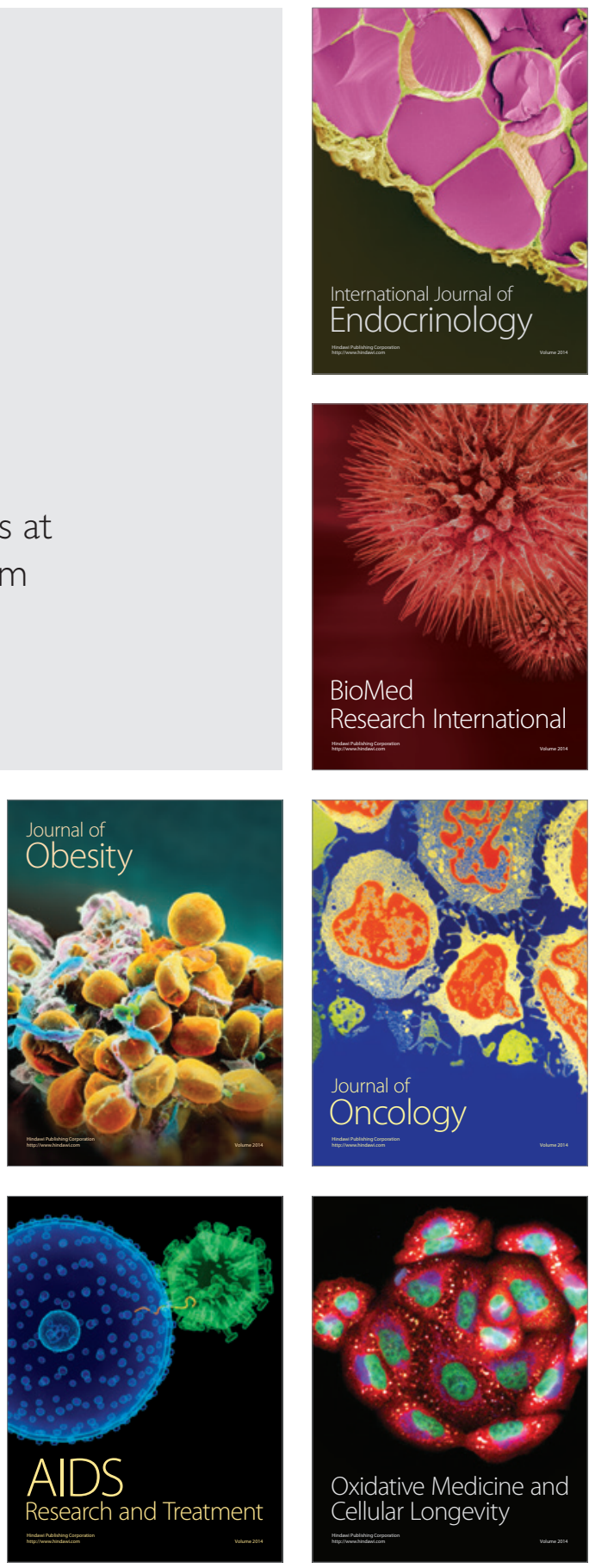